\title{
Feasibility and diagnostic accuracy of point-of-care handheld echocardiography in acute ischemic stroke patients - a pilot study
}

Peter Kraft ${ }^{1,3^{*}+}$, Anna Fleischer ${ }^{2 \dagger}$, Silke Wiedmann ${ }^{2,3}$, Viktoria Rücker ${ }^{2}$, Daniel Mackenrodt ${ }^{1,2,3}$, Caroline Morbach ${ }^{3,5}$, Uwe Malzahn'2, Christoph Kleinschnitz ${ }^{1,3,4}$, Stefan Störk ${ }^{3,5}$ and Peter U. Heuschmann 2,3

\begin{abstract}
Background: Standard echocardiography (SE) is an essential part of the routine diagnostic work-up after ischemic stroke (IS) and also serves for research purposes. However, access to SE is often limited. We aimed to assess feasibility and accuracy of point-of-care (POC) echocardiography in a stroke unit (SU) setting.

Methods: IS patients were recruited on the SU of the University Hospital Würzburg, Germany. Two SU team members were trained in POC echocardiography for a three-month period to assess a set of predefined cardiac parameters including left ventricular ejection fraction (LVEF). Diagnostic agreement was assessed by comparing POC with SE executed by an expert sonographer, and intraclass correlation coefficient (ICC) or kappa (K) with 95\% confidence intervals $(95 \% \mathrm{Cl})$ were calculated.

Results: In the 78 patients receiving both POC and SE agreement for cardiac parameters was good, with ICC varying from $0.82(95 \% \mathrm{Cl} 0.71-0.89)$ to 0.93 (95\% Cl 0.87-0.96), and $\mathrm{k}$ from $0.39(-95 \% \mathrm{Cl} 0.14-0.92)$ to $0.79(95 \% \mathrm{Cl}$ 0.67-0.91). Detection of systolic dysfunction with POC echocardiography compared to SE was very good, with an area under the curve of 0.99 (0.96-1.00). Interrater agreement for LVEF measured by POC echocardiography was good with $\mathrm{k} 0.63$ (95\% Cl 0.40-0.85).

Conclusions: POC echocardiography in a SU setting is feasible enabling reliable quantification of LVEF and preliminary assessment of selected cardiac parameters that might be used for research purposes. Its potential clinical utility in triaging stroke patients who should undergo or do not necessarily require SE needs to be investigated in larger prospective diagnostic studies.
\end{abstract}

Keywords: Ischemic stroke, Point-of-care echocardiography, Ejection fraction, Stroke unit, Systolic dysfunction, Feasibility, Accuracy

\section{Background}

About $30 \%$ of ischemic strokes are attributable to cardiac causes such as atrial fibrillation, patent foramen ovale, or other structural or rhythm abnormalities associated with systolic dysfunction [1]. In patients without

\footnotetext{
* Correspondence: kraft_p1@ukw.de

${ }^{\dagger}$ Equal contributors

'Department of Neurology, University Hospital of Würzburg, Josef-Schneider-Str. 11, 97080 Würzburg, Germany

${ }^{3}$ Comprehensive Heart Failure Center, University of Würzburg, Würzburg, Germany

Full list of author information is available at the end of the article
}

known atrial fibrillation, echocardiography is considered essential to determine the underlying cause of stroke in research studies and for guiding secondary prevention in clinical routine; its use, therefore, is recommended by current clinical guidelines [2-4]. However, access of stroke patients to echocardiography that is executed by an ultrasound specialist is often limited, which may delay diagnosis, extend hospital stay and increase healthcare costs [5]. Point-of-care (POC) echocardiography (so-called 'handheld echo') might be used in stroke patients as a screening tool for the detection of treatment 
relevant cardiac abnormalities, such as systolic dysfunction, aortic and mitral valve disease, left ventricular hypertrophy, atrial septal aneurysm, and pleural and pericardial effusion. In addition, POC echocardiography could contribute valuable information needed for research purposes, such as stroke etiology in diagnostic studies.

In this pilot study we investigated the diagnostic utility and accuracy of POC echocardiography in acute stroke patients performed by a non-expert examiner after a defined training phase on a stroke unit compared to a state-of-the-art transthoracic echocardiography performed by an expert sonographer under standardized cardiological conditions.

\section{Methods}

\section{Population}

Patients were recruited consecutively on the stroke unit of the University Hospital Würzburg between May and August 2014. The patient group is a sub-sample of the prospective SICFAIL (Stroke Induced Cardiac FAILure) cohort study evaluating the prevalence and time course of cardiac dysfunction in patients with ischemic stroke. Inclusion criteria were a diagnosis of acute ischemic stroke according to world health organization (WHO) definition [6], age $\geq 18$ years, and written informed consent.

\section{Echocardiography}

POC echocardiography was performed using a portable cardiac ultrasound device (Vivid q, GE Healthcare) equipped with a M3S $1.5-4.0 \mathrm{MHz}$ scanner (Additional file 1: Figure S1) in all study participants by a medical student (AF) specifically trained in this technique for the purpose of this study. A neurologist unfamiliar with echocardiography prior to study start (PK) received similar training to quantify inter-rater reliability. Training of the two non-cardiologist sonographers (AF, PK) was provided by expert sonographers of the Comprehensive Heart Failure Center Würzburg, spanned a period of about 3 months, included POC and standard echocardiography, and was completed via 3 certification scans 2 months prior to study initiation. For POC echocardiography measurements, patients were examined lying left-sided in their regular stroke unit beds, i.e. not on a dedicated echocardiography stretcher. The left arm had to be bended behind the head and the upper part of the bed was elevated by $20-30^{\circ}$ to the horizontal plane. The patients had to follow the examiner's breathing instructions as far as possible. The examination started with the parasternal and continued with the apical and finally the subcostal insonation.

In addition, all patients underwent a reference transthoracic standard echocardiography according to current guidelines performed on a high-end ultrasound device (Vivid E9, GE Healthcare; GE M5S-D matrix single-crystal phased array transducer) by an expert sonographer of the Comprehensive Heart Failure Center [7].

In every patient 3 electrocardiography-triggered heart cycles were stored for both the POC and standard echocardiography. Basic cardiac parameters were assessed by standard scanning procedures including left ventricular ejection fraction (LVEF), left heart dimensions, wall thickness, major valve abnormalities, and pericardial or pleural effusion. LVEF was calculated according to the Simpson's biplane method or, in case of suboptimal imaging conditions, according to the Simpson's monoplane method. An LVEF $<55 \%$ was defined as systolic dysfunction. Both scans, POC and standard echocardiography were performed within $48 \mathrm{~h}$. In every case, the examiner performing the POC test vs. the standard echocardiography was blinded to the results of the respectively other examination.

\section{Statistics and sample size calculation}

For continuous variables the mean and standard deviation, for categorical variables the frequencies and proportions were reported. To assess the agreement between the POC and standard echocardiography for qualitative variables, Cohen's kappa with its respective 95\% confidence interval (CI) was calculated. To assess the agreement between the POC and standard echocardiography for quantitative variables in the sense of conformity with standard echocardiography as reference, the intraclass correlation coefficient (ICC) for two raters (for continuous variables) with their respective 95\% CI were calculated.

The sample size estimation was based on LVEF as the main read-out parameter as the SICFAIL study focused on echocardiographic measurement of cardiac function defined by ejection fraction. Area under the receiver operation curve (AUC), sensitivity and specificity were assessed for the detection of systolic dysfunction with POC echocardiography; 78 were required to detect an AUC of 0.95 with the power of $80 \%$ to the significance level of 5\%. Analyses were performed with SPSS Software (SPSS 23, IBM Corp.).

\section{Ethical approval}

The study was approved from the University Hospital Würzburg Ethics Committee (AZ 176/13) and patients provided their written consent to participate in the study prior to any examination. The study was reported according to the Standards for Reporting Diagnostic accuracy studies (STARD) criteria. 


\section{Results}

Eighty one patients with ischemic stroke were recruited for this study. Three subjects (3.7\%) were excluded from analyses as they refused subsequent standard echocardiography. Hence, 78 patients provided two echocardiograms. In $76 \%$ of cases both echocardiographies were conducted within $24 \mathrm{~h}$, in $24 \%$ within $48 \mathrm{~h}$. In 56 patients $(72 \%)$ standard echocardiography followed POC examination, in 22 patients (28\%) POC echocardiography was conducted first. The demographic and clinical baseline characteristics are shown in Table 1 .

In a first step we focused on the measurement of LVEF being the main read-out parameter in our study. Of the 78 patients with two echocardiograms, reliable LVEF assessment was achieved in 73 patients, since ultrasound quality of both POC and standard echocardiography was insufficient in 5 patients; in these subjects, LVEF was assessed by eyeballing (i.e. $<55 \%$ or $\geq 55 \%$ ). In a second step, we investigated a series of other potentially treatment-relevant cardiac structures. Table 2 summarizes the quality of ultrasound conditions and results of echocardiographic measurements in POC and standard echocardiography.

Interrater reliability was calculated between POC and standard echocardiography for all measured parameters. ICC ranged from 0.82 for LVEF to 0.93 for left ventricular end-systolic diameter (Table 2). Agreement coefficients for cardiac measurements ranged from 0.39 (pleural effusion) to 0.69 (atrial septum aneurysm), and for sonographic conditions from 0.65 (apical view) to 0.79 (subcostal view) (Table 2).

Given the study design and rationale as well as the LVEF-specific sample size estimation we restricted the

Table 1 Demographical and baseline clinical data of patients

\begin{tabular}{ll}
\hline Age, years, mean (SD) & $68.0(13.5)$ \\
Male, n (\%) & $47(61.0)$ \\
Hypertension, n (\%) & $44(58.7)$ \\
Diabetes mellitus, n(\%) & $18(24.0)$ \\
Hyperlipidemia, n (\%) & $21(28.8)$ \\
Tobacco, n (\%) & $13(17.1)$ \\
Atrial Fibrillation, n (\%) & $5(6.8)$ \\
Angina Pectoris, n (\%) & $6(8.1)$ \\
History of myocardial infarction, n (\%) & $4(5.4)$ \\
History of heart failure, n (\%) & $3(4.2)$ \\
Peripheral artery disease, n (\%) & $5(6.8)$ \\
NIHSS at admission, median (IQR) & $3(1-4)$ \\
Systolic blood pressure, mmHg, mean (SD) & $148.7(22.0)$ \\
Diastolic blood pressure, mmHg, mean (SD) & $77.4(14.6)$ \\
Body mass index, kg/m², mean (SD) & $27.5(5.1)$ \\
\hline
\end{tabular}

$S D$ standard deviation, IQR interquartile range NIHSS National Institutes of Health Stroke Scale score calculation of further statistical values to LVEF as a marker for systolic function. Sensitivity of POC echocardiography for detecting systolic dysfunction was $100 \%$, specificity 92.6\%, and AUC 0.99 (95\% CI, 0.96-1.00). Interrater reliability of the LVEF measurement by POC echocardiography between the two non-expert examiners was analyzed by an independent investigation of 18 patients. The ICC between the two raters for LVEF was 0.64 (95\% CI 0.26-0.85).

\section{Discussion}

This pilot study showed that POC echocardiographic assessment performed in the ambitious setting of a stroke unit by stroke unit staff using a portable device is feasible and achieves reliable results regarding selected key characteristics of cardiac dimensions and function.

Feasibility and reliability of POC echocardiography have been assessed in different settings before. A recent report underscores the feasibility and usefulness to establish POC echocardiography even during the medical studies [8]. Our results are in line with previous investigations reporting good correlations between POC and standard echocardiography for the quantification of cardiac structures and functions in patients with suspected cardiac abnormalities (e.g. congestive heart failure) treated in cardiology departments [8,9]. Of note, in most studies ultrasound examiners were experienced cardiologists and/or sonographers [4, 9-11], whereas in our study non-cardiologists performed the POC examinations. Another group found only moderate correlations between POC and standard echocardiography, and discordant findings (mainly regarding wall motion abnormalities) in $27 \%$ of adult out-patients even when performed and interpreted by experienced operators [11].

To the best of our knowledge, we present the first report evaluating portable echocardiography in patients with acute ischemic stroke in the setting of a stroke unit. The majority of patients had neurological deficits and POC echocardiography had to be performed in regular hospital beds which made the examination particularly challenging. Nevertheless, we were able to demonstrate high precision for the detection of systolic dysfunction and structural cardiac abnormalities compared to standard echocardiography performed by expert sonographers under routine conditions.

POC echocardiography might be a useful tool for documenting relevant cardiac abnormalities, used to inform research studies regarding the underlying cause of stroke. The role of POC echocardiography regarding intraventricular thrombus or other life threatening findings could not be assessed as they were not prevalent in our patient collective. Nevertheless, in this pilot study, atrial septal aneurysms could be detected with high precision. Further studies to evaluate the reliability of 
Table 2 Point-of-care and standard echocardiographic data

\begin{tabular}{|c|c|c|c|c|}
\hline & $\begin{array}{l}\text { Point-of-care } \\
\text { echocardiography }\end{array}$ & $\begin{array}{l}\text { Standard } \\
\text { echocardiography }\end{array}$ & $\begin{array}{l}\text { Intraclass correlation } \\
\text { coefficient } \\
(95 \% \mathrm{Cl})\end{array}$ & $\begin{array}{l}\text { Agreement } \\
\text { coefficient k } \\
(95 \% \mathrm{Cl})\end{array}$ \\
\hline \multicolumn{5}{|l|}{ Image quality, n (\%) } \\
\hline Parasternal & & & & $0.73(0.61-0.86)$ \\
\hline Good & $35(45.5)$ & $40(51.3)$ & & \\
\hline Moderate & $22(28.6)$ & $20(25.6)$ & & \\
\hline Poor & $20(26.0)$ & $18(23.1)$ & & \\
\hline Apical & & & & $0.65(0.50-0.79)$ \\
\hline Good & $32(42.1)$ & $39(50.0)$ & & \\
\hline Moderate & $26(34.2)$ & $25(32.1)$ & & \\
\hline Poor & $18(23.7)$ & $14(17.9)$ & & \\
\hline Subcostal & & & & $0.79(0.67-0.91)$ \\
\hline Good & $38(48.7)$ & $41(52.6)$ & & \\
\hline Moderate & $28(35.9)$ & $24(30.8)$ & & \\
\hline Poor & $12(15.4)$ & $13(16.7)$ & & \\
\hline Left ventricular ejection fraction, \%, mean (SD) & $60.8(7.2)$ & $62.3(5.6)$ & $0.82(0.71-0.89)$ & \\
\hline Left ventricular ejection fraction, n (\%) & & & & $0.63(0.40-0.85)$ \\
\hline$<30 \%$ & $0(0.0)$ & $0(0.0)$ & & \\
\hline $30-44 \%$ & $3(3.9)$ & $1(1.3)$ & & \\
\hline $45-55 \%$ & $10(13.2)$ & $7(9.1)$ & & \\
\hline$>55 \%$ & $63(82.9)$ & 69 (89.6) & & \\
\hline Not measurable, $n$ & 2 & 1 & & \\
\hline Left ventricular end-diastolic wall thickness (septum), mm, mean (SD) & $10.8(1.7)$ & $10.7(1.7)$ & $0.84(0.76-0.90)$ & \\
\hline $\begin{array}{l}\text { Left ventricular end-diastolic wall thickness (posterior wall) mm, } \\
\text { mean (SD) }\end{array}$ & $10.5(1.7)$ & $10.0(1.7)$ & $0.85(0.72-0.92)$ & \\
\hline Left ventricular end-systolic diameter, mm, mean (SD) & $33.7(6.4)$ & $32.9(7.1)$ & $0.93(0.87-0.96)$ & \\
\hline Left ventricular end-diastolic diameter, mm, mean (SD) & $49.8(5.5)$ & $50.0(5.5)$ & $0.86(0.79-0.91)$ & \\
\hline Aortic valve maximal systolic flow velocity, m/s, mean (SD) & $1.5(0.4)$ & $1.5(0.4)$ & $0.92(0.88-0.95)$ & \\
\hline Pericardial effusion, n (\%) & $0(0.0)$ & $0(0.0)$ & & $0.00(0.00-0.00)$ \\
\hline Pleural effusion, $\mathrm{n}(\%)$ & $1(1.3)$ & $1(1.3)$ & & $0.39(-0.14-0.92)$ \\
\hline Atrial septum aneurysm, n (\%) & $3(3.9)$ & $4(5.1)$ & & $0.69(0.39-0.98)$ \\
\hline Heart rate during examination, 1/min, mean (SD) & $69.8(10.7)$ & $69.7(11.9)$ & $0.67(0.52-0.78)$ & \\
\hline
\end{tabular}

$\mathrm{Cl}$ confidence interval, $S D$ standard deviation

detection of persistent foramen ovale via transthoracic POC echocardiography applying right heart contrast agent seem promising and might make standard echocardiography unnecessary in certain patients.

Some limitations of the current pilot study need to be addressed. As it was mainly designed for measurement of LVEF in the SICFAIL study, we did not include all potentially treatment-relevant cardiac parameters into the echocardiography protocol (e.g. detailed description of regional LV dysfunction or mitral valve analysis). The sample size estimation was calculated for measurement of LVEF as a marker of systolic function only. Therefore, despite high accuracy of POC measurements, low patient numbers with peculiar cardiologic findings (pericardial effusion $n=0$, pleural effusion $n=1$, atrial septum aneurysm $n=4$, intracardiac thrombus $n=0$ ) impede reliable conclusions regarding these conditions. Due to the monocentric design, generalization of our results to conditions outside of our hospital is limited. The echocardiography device was of high quality and of the same brand as the reference device; this might have improved comparability and reliability of measurements. The stroke unit setting (e.g. type of hospital beds, illumination of patient rooms) may also vary amongst institutions. In addition, potential selection bias cannot be excluded as severely handicapped patients could not provide informed consent. 


\section{Conclusion}

POC echocardiography is feasible in an acute stroke unit setting. Given the high accuracy for the detection of systolic dysfunction, it may be used for research purposes evaluating cardiac function after stroke. Its further clinical role as a screening tool for guiding individual patient treatment and management, such as fluid management in the acute situation, or for guiding secondary prevention, e.g. by detection of a cardiac source of embolism, which could render a standard echocardiogram unnecessary under certain conditions, needs to be established in larger prospective diagnostic studies with a sufficient number of patients with peculiar cardiologic findings.

\section{Additional file}

Additional file 1: Figure S1. Illustration of the point-of-care ultrasound device (Vivid q, GE Healthcare, equipped with a M3S 1.5-4.0 MHz scanner). (DOCX $36 \mathrm{~kb}$ )

\begin{abstract}
Abbreviations
AUC: Area under curve; Cl: Confidence interval; ICC: Intraclass correlation coefficient; IQR: Interquartile range; IS: Ischemic stroke; LVEF: Left ventricular ejection fraction; NIHSS: National Institutes of Health Stroke Scale score; POC: Point of care; SD: Standard deviation; SE: Standard echocardiography; SICFAIL: Stroke induced cardiac failure; SU: Stroke unit; WHO: World health organization
\end{abstract}

\section{Acknowledgements}

The authors thank Martina Bauer, Elisabeth Nau, and Jasmin Simon for providing training and echocardiography examinations.

\section{Funding}

This work was supported by the Federal Ministry of Education and Research within the Comprehensive Heart Failure Center Würzburg (grant 01E01504) The funder did not influence the design of the study, the data collection, the analysis and interpretation of data and the writing of the manuscript.

\section{Availability of data and materials}

All necessary data generated or analyzed during this study are included in this published article (results section, tables). Individual patient data that support the findings of this analysis are available upon request from the corresponding author (PK) in strict accordance to local data security restrictions.

\section{Authors' contributions}

PK and AF performed POC echocardiography examinations; PK, AF, SW, VR, CM, UM, SS and PUH statistically analyzed and interpreted data; PK, AF, SW, CM, SS and PUH drafted the manuscript; after that all authors (including DM and $(\mathrm{K})$ contributed in revision of the manuscript for important intellectual content; all authors (including DM and CK) read and approved the final manuscript.

\section{Authors' information}

Not applicable

\section{Ethics approval and consent to participate}

The study was approved from the University Hospital Würzburg Ethics Committee (AZ 176/13) and patients provided their written consent to participate in the study prior to any examination.

\section{Competing interests}

PK received honoraria for advisory boards and travel grants from BoehringerIngelheim, Bayer, Daiichi-Sankyo and Bristol-Myers Squibb. SW receives research support from the German Ministry of Research and Education and Deutsche Herzstiftung e.V.. PUH reports grants from German Ministry of Research and Education, European Union, Charité, Berlin Chamber of Physicians, German Parkinson Society, University Hospital Würzburg, RobertKoch-Institute, German Heart Foundation, Charité-Universitätsmedizin Berlin (within Mondafis; supported by an unrestricted research grant to the Charité from Bayer), University Göttingen (within FIND-AF-randomized; supported by an unrestricted research grant to the University Göttingen from BoehringerIngelheim), University Hospital Heidelberg (within RASUNOA-prime; supported by an unrestricted research grant to the University Hospital Heidelberg from Bayer, BMS, Boehringer-Ingelheim, Daiichi Sankyo), outside the submitted work

\section{Publisher's Note}

Springer Nature remains neutral with regard to jurisdictional claims in published maps and institutional affiliations.

\section{Author details}

${ }^{1}$ Department of Neurology, University Hospital of Würzburg, Josef-Schneider-Str. 11, 97080 Würzburg, Germany. ${ }^{2}$ Institute of Clinical Epidemiology and Biometry, University of Würzburg, Würzburg, Germany. ${ }^{3}$ Comprehensive Heart Failure Center, University of Würzburg, Würzburg, Germany. ${ }^{4}$ Department of Neurology, University Hospital Essen, Essen, Germany. ${ }^{5}$ Department of Internal Medicine, University Hospital Würzburg, Würzburg, Germany.

Received: 28 April 2017 Accepted: 3 August 2017

Published online: 11 August 2017

\section{References}

1. Kolominsky-Rabas PL, Weber M, Gefeller O, Neundoerfer B, Heuschmann PU Epidemiology of ischemic stroke subtypes according to TOAST criteria: incidence, recurrence, and long-term survival in ischemic stroke subtypes: a population-based study. Stroke. 2001:32:2735-40.

2. Herm J, Konieczny M, Jungehulsing GJ, et al. Should transesophageal echocardiography be performed in acute stroke patients with atrial fibrillation? J Clin Neurosci. 2013;20:554-9.

3. Kernan WN, Ovbiagele B, Black HR, et al. Guidelines for the prevention of stroke in patients with stroke and transient ischemic attack: a guideline for healthcare professionals from the American Heart Association/American Stroke Association. Stroke. 2014:45:2160-236.

4. Ringleb PA, Bousser MG, Ford G, Bath P, Brainin M, Caso V, et al. Guidelines for management of ischaemic stroke and transient ischaemic attack 2008. Cerebrovasc Dis. 2008;25:457-507.

5. Trambaiolo P, Papetti F, Posteraro A, et al. A hand-carried cardiac ultrasound device in the outpatient cardiology clinic reduces the need for standard echocardiography. Heart. 2007:93:470-5.

6. Aho K, Harmsen P, Hatano S, Marquardsen J, Smirnov VE, Strasser T. Cerebrovascular disease in the community: results of a WHO collaborative study. Bull World Health Organ. 1980;58:113-30.

7. Lang RM, Bierig M, Devereux RB, et al. Recommendations for chamber quantification. Eur J Echocardiogr. 2006;7:79-108.

8. Ho AM, Critchley LA, Leung JY, et al. Introducing final-year medical students to pocket-sized ultrasound imaging: teaching transthoracic echocardiography on a 2-week anesthesia rotation. Teach Learn Med. 2015;27:307-13.

9. Khan HA, Wineinger NE, Uddin PQ, Mehta HS, Rubenson DS, Topol EJ. Can hospital rounds with pocket ultrasound by cardiologists reduce standard echocardiography. Am J Med. 2014;127:669. e1-7

10. Andersen GN, Haugen BO, Graven T, Salvesen O, Mjolstad OC, Dalen H. Feasibility and reliability of point-of-care pocket-sized echocardiography. Eur J Echocardiogr. 2011;12:665-70.

11. Cullen MW, Blauwet LA, Vatury OM, et al. Diagnostic capability of comprehensive handheld vs transthoracic echocardiography. Mayo Clin Proc. 2014;89:790-8. 